\title{
The Impact of Bulk Defects, Surface States, and Excitons on Yellow and Ultraviolet Photoluminescence in GaN
}

\author{
M. MATYS $^{a, *}$, M. MiCZEK $^{a}$, B. AdAMOWICZ ${ }^{a}$, Z.R. ŻYTKIEWICZ ${ }^{b}$, E. KAMiŃSKA $^{c}$, \\ A. PiOtrowska ${ }^{c}$ AND T. HAShizUme ${ }^{d}$ \\ ${ }^{a}$ Department of Surface Physics and Nanostructures, Institute of Physics - Centre for Science and Education \\ Silesian University of Technology, Bolesława Krzywoustego 2, 44-100 Gliwice, Poland \\ ${ }^{b}$ Institute of Physics, Polish Academy of Sciences, al. Lotników 32/46, 02-668 Warsaw, Poland \\ ${ }^{c}$ Institute of Electron Technology, al. Lotników 32/46, 02-668 Warsaw, Poland \\ ${ }^{d}$ Research Center for Integrated Quantum Electronics, Hokkaido University \\ Kita-13 Nishi-8, Sapporo 060-8628, Japan
}

\begin{abstract}
The quantitative analysis of the influence of deep bulk levels, surface states and excitons on yellow, green and ultraviolet photoluminescence from $n$-type GaN was performed. The theoretical calculations of recombination rates in the bulk and at $n$-GaN surface versus UV-excitation intensity were done numerically using finite element method basing on drift-diffusion model assuming point deep levels, continuous energetic distribution of surface states, as well as excitons. The obtained results of the photoluminescence intensity were compared with experimental data (measured within the range from $10^{15}$ to $10^{19}$ photon $\mathrm{cm}^{-2} \mathrm{~s}^{-1}$ ) for $n$-GaN samples with various surface passivating layers $\left(\mathrm{Al}_{2} \mathrm{O}_{3}, \mathrm{SiO}_{2}\right)$.
\end{abstract}

PACS: 78.55.-m, 71.35.-y, 73.20.-r, 71.55.-i, 02.70.Dh

\section{Introduction}

Gallium nitride due to its unique properties, like wide bandgap, chemical, electrical, and thermal hardness, is widely applied in optoelectronic devices, e.g. lasers and photodetectors. However, even high-quality GaN samples still contain a lot of point defects and surface states which diminish the optical efficiency and shorten the lifetime of the devices.

The room temperature photoluminescence (PL) spectra of GaN samples exhibit, first of all, the peak related to near-band transitions and free-exciton recombination in the UV range and also, in most cases, a wide peak in the region from 2.2 to $2.5 \mathrm{eV}$, i.e. so-called yellow luminescence and green luminescence (YGL), which is attributed to the transitions from shallow donor levels to deep acceptor ones. It has been recently reported that these deep acceptor centers are gallium vacancy-oxygen-in-nitrogen-site $\left(\mathrm{V}_{\mathrm{Ga}}-\mathrm{O}_{\mathrm{N}}\right)$ defect complexes [1-3] which appear during GaN growth.

Another important factor in PL analysis is the surface states which are usually neglected or analyzed in simplified way using unrealistic energetic distributions [4] whereas it is known from the literature that the surface processing can change PL intensity from a semiconductor by 3 orders of magnitude [5].

The third contribution to PL in GaN is a free-exciton recombination, which gives rise to photon emission and appearance of a well developed peak in the PL spectra

* corresponding author; e-mail: Maciej.Matys@polsl.pl even at room temperature because the exciton binding energy is much larger in GaN than in the other III-V semiconductors [6]. Therefore, it is evident that quantitative analysis of PL from GaN, particularly in context of electronic properties of GaN-based structures, is a difficult task and remains under wide debate [7].

In this work, we made an attempt to analyze theoretically the influence of various factors on both bulk and surface recombination processes in $n$-GaN, i.e. the bulk point defects, surface states and excitons on yellow-green and ultraviolet channels of PL versus excitation light intensity $\Phi$ in a wide range from $10^{8}$ to $10^{24}$ photon $\mathrm{cm}^{-2} \mathrm{~s}^{-1}$. The calculation results, obtained numerically from a drift-diffusion model by using finite element method, were compared with PL experimental data from $n$-GaN samples with various surface passivating layers $\left(\mathrm{Al}_{2} \mathrm{O}_{3}, \mathrm{SiO}_{2}\right)$.

\section{Experiment}

We studied two GaN samples fabricated on sapphire by metal-organic chemical vapor deposition (MOCVD) and molecular beam epitaxy (MBE), respectively. The free electron concentrations at room temperature were determined from the Hall measurements as $4.5 \times 10^{17} \mathrm{~cm}^{-3}$ and $10^{17} \mathrm{~cm}^{-3}$, respectively. The former sample was covered by $20 \mathrm{~nm}$ thick $\mathrm{Al}_{2} \mathrm{O}_{3}$ film deposited by atomic layer deposition (ALD) technique whereas the latter one by $70 \mathrm{~nm}$ thick $\mathrm{SiO}_{2}$ layer obtained by plasma-enhanced chemical vapor deposition (PECVD) process. The PL measurements were done using $\mathrm{He}-\mathrm{Cd}$ laser with $325 \mathrm{~nm}$ line and maximum power of $200 \mathrm{~mW}$ and CCD detector with IR-VIS-UV triple grating monochromator. 


\section{Theoretical model}

In the calculations, the semi-infinite GaN sample with shallow donor (dopant-related) levels with the concentration $N_{\mathrm{d}}\left(10^{17}\right.$ and $\left.4.5 \times 10^{17} \mathrm{~cm}^{-3}\right)$ and deep acceptor-type point defects with the typical density $N_{\mathrm{a}}\left(4.5 \times 10^{15}\right.$ and $8 \times 10^{15} \mathrm{~cm}^{-3}$ ) was assumed [8]. Under excitation by photons with energy above the GaN bandgap $(3.4 \mathrm{eV})$, electron-hole pairs are generated in GaN. Some holes are captured by ionized acceptor defects and then the captured holes can recombine radiatively with electrons from the conduction band. At the same time, free electrons and holes can create free excitons or recombine with photon emission (the band-to-band transition). We assumed that the recombination of the free excitons can be radiative and nonradiative. Due to thermal excitation, the holes captured by defects can jump to the valence band whereas the excitons can decay into free electrons and holes.

The nonradiative bulk recombination was taken into account by using the Shockley-Read-Hall (SRH) model $[9,10]$. The carrier transport was described by classical drift-diffusion model in the steady state case and at room temperature with the boundary conditions determined by surface recombination and surface charge [11, 12]. The U-shaped energetic distribution of the surface state density, $N_{\mathrm{ss}}(E)$, was assumed in accordance with disorder induced gap state (DIGS) model [13] with the minimum of $N_{\mathrm{ss}}(E)$ curve, $N_{\mathrm{ss} 0}=10^{11}$ and $5 \times 10^{12} \mathrm{eV}^{-1} \mathrm{~cm}^{-2}$. The model equations were solved numerically using a finite element method in the wide range of excitation light intensity (in terms of photon flux density), $\Phi$, from $10^{8}$ to $10^{24}$ photon $\mathrm{cm}^{-2} \mathrm{~s}^{-1}$.

\section{Results and discussion}

The typical PL spectra measured for two different $n$-GaN samples under excitation of $2.5 \times 10^{18}$ photon $\mathrm{cm}^{-2} \mathrm{~s}^{-1}$ are summarized in Fig. 1. It is evident that both samples emit light in YGL region $(2.2-2.5 \mathrm{eV})$ as well as in ultraviolet one, i.e. near band edge (NBE) at about $3.4 \mathrm{eV}$. It should be noted that the PL signals from the two samples differ from each other by about 2000 times in the whole range of excitation intensity.

In order to understand the observed PL features all fundamental recombination channels were analyzed numerically and the results of those simulations were summarized in Figs. 2 and 3.

Figure $2 \mathrm{a}$ presents the dependence of the ratio of the radiative recombination rate of free excitons $(\mathrm{FE})$ to that one of the band-to-band (BB) transition versus $\Phi$ for different doping level, $N_{\mathrm{d}}$. Both processes contribute to NBE peak of PL spectrum but their ratio is still under debate in the literature [14]. From our calculation, it seems that for lowly doped GaN, the FE transition is more efficient than the $\mathrm{BB}$ one whereas the $\mathrm{BB}$ channel becomes comparable to the FE one for $N_{\mathrm{d}}=4.5 \times 10^{17} \mathrm{~cm}^{-3}$. This effect is probably caused by the stronger screening of the excitons by more free carriers. The influence of the surface states on the $\mathrm{FE} / \mathrm{BB}$ ratio can be neglected in this

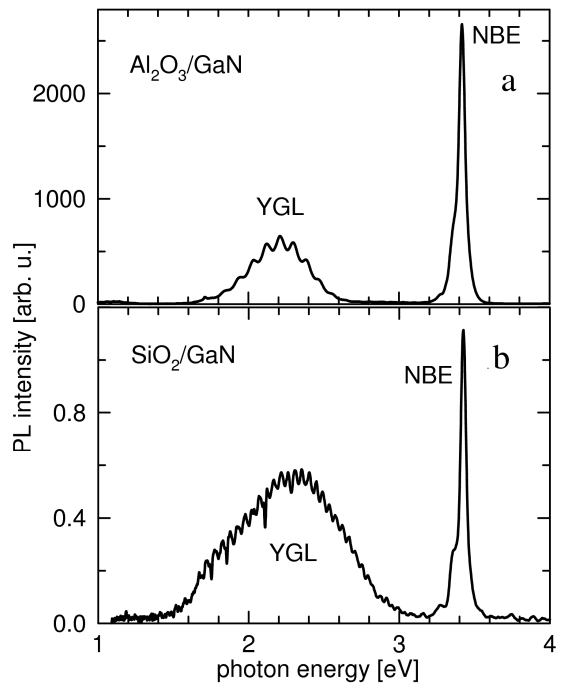

Fig. 1. Photoluminescence spectra measured from $n$-GaN samples on sapphire passivated by (a) $\mathrm{Al}_{2} \mathrm{O}_{3}$ layer deposited by ALD, (b) $\mathrm{SiO}_{2}$ deposited by PECVD. The excitation light intensity $\Phi=2.5 \times 10^{18}$ photon $\mathrm{cm}^{-2} \mathrm{~s}^{-1}$

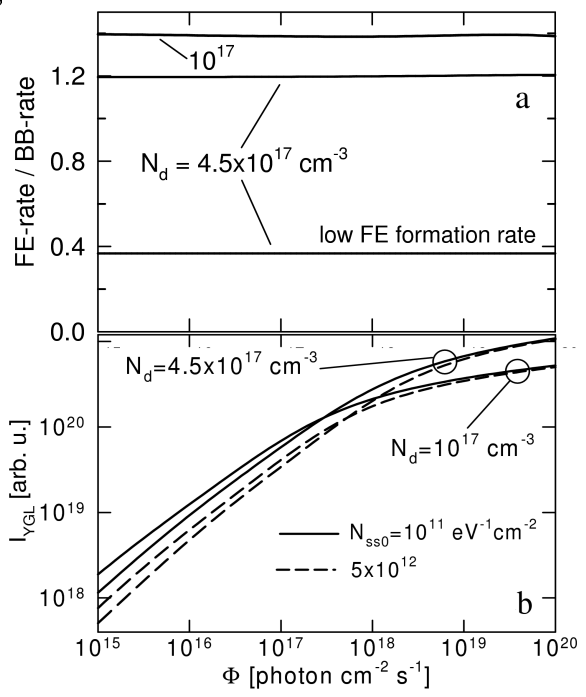

Fig. 2. The dependences of (a) the ratio of the radiative recombination rate of free excitons to that one of the band-to-band transition and (b) the yellow-and-green luminescence intensity, $I_{\mathrm{YGL}}$, versus excitation light intensity, $\Phi$, for the doping levels, $N_{\mathrm{d}}, 10^{17}$ and $4.5 \times 10^{17} \mathrm{~cm}^{-3}$ and the minimum density of surface states, $N_{\mathrm{ss} 0}, 10^{11}$ and $5 \times 10^{12} \mathrm{eV}^{-1} \mathrm{~cm}^{-2}$.

case. The most important point is that the $\mathrm{FE} / \mathrm{BB}$ ratio is practically independent of $\Phi$.

In Fig. 2b, the dependence of YGL intensity versus excitation light intensity for the same set of parameters like in Fig. $2 \mathrm{a}$ is presented. The change of the curve slope due to saturation of the point defects versus excitation power as the recombination centers is well noticeable. It is also obvious that the surface states have significant impact on YGL and their neglect during the determination of ra- 


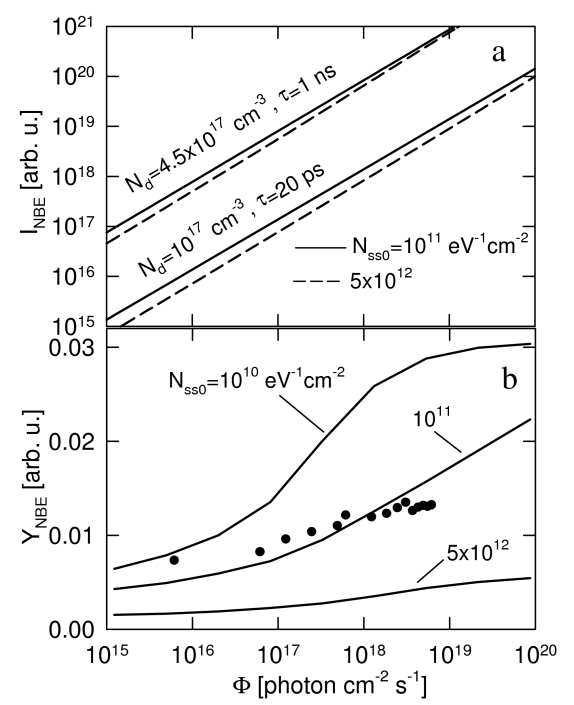

Fig. 3. (a) The dependences of near band edge luminescence, $I_{\mathrm{NBE}}$, versus excitation light intensity, $\Phi$, for the doping levels, $N_{\mathrm{d}}, 10^{17}$ and $4.5 \times 10^{17} \mathrm{~cm}^{-3}$, the minimum density of surface states, $N_{\mathrm{ss} 0}, 10^{11}$ and $5 \times 10^{12} \mathrm{eV}^{-1} \mathrm{~cm}^{-2}$, and various nonradiative lifetimes of excitons, $\tau$. (b) The calculated curves of near band edge PL efficiency, $Y_{\mathrm{NBE}}$, versus excitation light intensity, $\Phi$, for the various values of the minimum density of surface states, $N_{\mathrm{ss} 0}$, compared to the experimental data (points) measured from the $\mathrm{Al}_{2} \mathrm{O}_{3} / \mathrm{GaN}$ sample.

diative defects from PL spectra [4] can lead to erroneous results, especially for lowly doped GaN. The impact of surface states is also significant in the case of NBE peak as shown in Fig. 3a. However, from our calculations, it appears that the increase in the surface state density, $N_{\mathrm{ss} 0}$, in the bulk defect concentration, $N_{\mathrm{a}}$, as well as the change of the SRH lifetime and other parameters can reduce NBE PL intensity (for the same value of $\Phi$ ) by at most 500 times which is still lower value than about 2000 observed in our experiment for two investigated samples.

In order to explain the experimental results it seems necessary to introduce another nonradiative recombination channel into the model. Our hypothesis is that it is a nonradiative recombination of free excitons [15]. After assuming the nonradiative lifetime of $\mathrm{FE}$ at the value of 20 ps [16] we obtain the NBE PL quenching by about 2000 times (Fig. 3a) which is in agreement with the experimental results.

Another possibility to explain this PL intensity change is nonradiative transitions via deep donor levels [3]. Both effects will be studied in more detail in future.

Finally, taking into account all the discussed recombination channels we calculated the quantum efficiency of the NBE PL as a function of $\Phi$ for different values of surface state density (in terms of $N_{\mathrm{ss} 0}$ ) and compared the obtained curves with the experimental results for the sample passivated with $\mathrm{Al}_{2} \mathrm{O}_{3}$ layer, as summarized in Fig. 3b. It is evident that our model proves the large role of surface states in PL mechanism and well repro- duces the evolution of the measured NBE PL quantum efficiency. The presented analysis shows the importance of the efficient passivation of the GaN surface to avoid the dramatic reduction of PL intensity.

\section{Conclusions}

The numerical analysis of the influence of bulk point defects, surface states and excitons on yellow-green and ultraviolet channels of PL versus excitation light intensity (in the range from $10^{8}$ to $10^{24}$ photon $\mathrm{cm}^{-2} \mathrm{~s}^{-1}$ ) was performed. The dependences of various recombination channel rates versus $\Phi$ were calculated from a drift-diffusion model by using finite element method and discussed in context of the measured room-temperature PL response from two differently passivated $\left(\mathrm{Al}_{2} \mathrm{O}_{3}, \mathrm{SiO}_{2}\right)$ $n$-GaN samples. In particular, the NBE PL quantum efficiency dependences simulated for different values of the surface state density were compared with the experimental ones. The obtained results proved the marked contribution of the surface states to recombination mechanisms giving rise to the possible strong reduction of PL in GaN-based structures.

\section{Acknowledgments}

The work was partially supported by InTechFun project of European Union Structural Funds in Poland (UDA-POIG.01.03.01-00-159/08).

\section{References}

[1] M.A. Reschnikov, H. Morkoç, J. Appl. Phys. 97, 061301 (2005).

[2] J. Neugebauer, C.G. Van de Walle, Appl. Phys. Lett. 69, 503 (1996).

[3] A. Sedhain, J. Li, J.Y. Lin, H.X. Jiang, Appl. Phys. Lett. 96, 151902 (2010).

[4] M.A. Reshchikov, Appl. Phys. Lett. 88, 202104 (2006).

[5] B.J. Skromme, C.J. Sandroff, E. Yablonovitch, T. Gmitter, Appl. Phys. Lett. 51, 2022 (1987).

[6] K.P. Korona, Phys. Rev. B 65, 235312 (2002).

[7] Nitride Semiconductor Devices. Principles and Simulation, Ed. J. Piprek, Wiley-VCH, Weinheim 2007.

[8] M.A. Reshchikov, R.Y. Korotkov, Phys. Rev. B 64, 115205 (2001).

[9] W. Shockley, W.T. Read, Phys. Rev. 87, 835 (1952).

[10] R.N. Hall, Phys. Rev. 87, 387 (1952).

[11] S. Selberherr, Analysis and Simulations of Semiconductor Devices, Springer, Wien 1998.

[12] B. Adamowicz, H. Hasegawa, Jpn. J. Appl. Phys. 37, 1631 (1998)

[13] H. Hasegawa, H. Ohno, J. Vac. Sci. Technol. B 4, 1130 (1986).

[14] M. Smith, J.Y. Lin, H.X. Jiang, M.A. Khan, Appl. Phys. Lett. 71, 635 (1997).

[15] S. Pau, J. Kuhl, M.A. Khan, C.J. Sun, Phys. Rev. B 58, 12916 (1998).

[16] F. Ishikawa, H. Hasegawa, Phys. Status Solidi C 0, 2707 (2003) 